\title{
FEATURE LINE BASED BUILDING DETECTION AND RECONSTRUCTION FROM OBLIQUE AIRBORNE IMAGERY
}

\author{
Quansheng Zhu, Wanshou Jiang, Jing Zhang
}

State Key Laboratory of Information Engineering in Surveying, Mapping and Remote Sensing, Wuhan University, 129

Luoyu Road, Wuhan, Hubei 430079, China - zhuqs@whu.edu.cn

KEY WORDS: Feature line, Building Detection, Building Reconstruction, Oblique Airborne Imagery

\begin{abstract}
:
In this paper, a feature line based method for building detection and reconstruction from oblique airborne imagery is presented. With the development of Multi-View Stereo technology, increasing photogrammetric softwares are provided to generate textured meshes from oblique airborne imagery. However, errors in image matching and mesh segmentation lead to the low geometrical accuracy of building models, especially at building boundaries. To simplify massive meshes and construct accurate 3D building models, we integrate multi-view images and meshes by using feature lines, in which contour lines are used for building detection and straight skeleton for building reconstruction. Firstly, through the contour clustering method, buildings can be quickly and robustly detected from meshes. Then, a feature preserving mesh segmentation method is applied to accurately extract 3D straight skeleton from meshes. Finally, straight feature lines derived from multi-view images are used to rectify inaccurate parts of 3D straight skeleton of buildings. Therefore, low quality model can be refined by the accuracy improvement of mesh feature lines and rectification with feature lines of multi-view images. The test dataset in Zürich is provided by ISPRS/EuroSDR initiative Benchmark on High Density Image Matching for DSM Computation. The experiments reveal that the proposed method can obtain convincing and high quality $3 \mathrm{D}$ building models from oblique airborne imagery.
\end{abstract}

\section{INTRODUCTION}

\subsection{Motivation \& Goals}

In recent years, oblique photography technology has been widely used in $3 \mathrm{~d}$ building modeling. With the advantage of having more facade information, oblique airborne imagery makes 3D building model more complete and realistic. In addition, the Multi-View Stereo (MVS) technology currently can provide dense, accurate point clouds and high quality $3 \mathrm{D}$ meshes. There are increasing photogrammetric softwares, such as Smart3DCapture by Acute3D and Photoscan by Agisoft, providing the automatic workflow to turn photos into high resolution 3D models. Although textured meshes have good visualization effect, it's also necessary to simplify massive surface meshes of complex, large-scale scenes, and construct $3 \mathrm{~d}$ semantic city models for advanced applications such as urban planning, indoor/outdoor pedestrian navigation, or cultural heritage.

Because of the complexity and diversity within the urban scene, it's easy to generate errors in the camera calibration and dense image matching, which could produce noisy and inaccurate point clouds comparing to LiDAR data. Moreover, to simplify raw model and generate meaningful levels of details, the significant step is planar patches extraction that also causes plane fitting errors. Therefore, the final constructed $3 \mathrm{D}$ building model always have low geometrical accuracy, especially at the boundary of buildings.

In order to solve the problem of low quality modeling from unreliable point clouds or meshes, it's necessary to combine images to refine the coarse model. Similarly, many researches integrate aerial imagery and LiDAR point clouds to obtain refined models(Chen, 2004; Cheng, 2011; Zhang, 2014). In these methods, feature line is used as an important feature to link imagery and point clouds. After main structure lines of roofs have been reconstructed from point clouds, these 3D feature lines can be projected to images and refined by 2D feature lines extracted from imagery. However, the automatic registration of imagery and point clouds is still a problem, while point clouds or meshes generated by MVS technology has intrinsic relation with original imagery, which greatly benefit the integration of imagery and MVS result.

Given that feature line is an easy and effective way to represent building structure from $2 \mathrm{D}$ imagery and $3 \mathrm{D}$ meshes, we choose it as the major tool for building detection and reconstruction from oblique airborne imagery. Contour line is one type of 3D feature line that has the characteristic of closed, having explicit topological relationships among each other, and distinguishable between buildings and other objects. These advantages make contours applicable in building detection from 3D meshes. Straight skeleton, such as ridge and valley lines, is the other type of feature line that provides powerful shape descriptor in building reconstruction. Through the space transformation, 2D feature lines derived from imagery can be used to optimize 3D straight skeleton constructed from meshes.

In this paper, we propose a feature line based method for building detection and reconstruction from oblique airborne imagery. Before our work, 3D surface meshes have been generated from multi-view images. Firstly, through the contour clustering method, buildings can be quickly and robustly detected from meshes. Then, a feature preserving mesh segmentation method is applied to accurately extract 3D straight skeleton from meshes. Finally, straight feature lines derived from multi-view images are used to rectify inaccurate parts of 3D straight skeleton of buildings. Therefore, low quality model caused by mesh segmentation errors and image matching errors can be separately refined by the accuracy improvement of mesh feature lines and rectification with feature lines of multi-view images.

\subsection{Related work}

\subsubsection{Building Detection from Oblique Imagery}

There are various researches for 3D building detection from oblique airborne imagery. The major difference of these methods is in the principal component of multi-source data that consists of original imagery and its derivatives, including 3D point clouds and meshes.

Xiao et al. (2012) proposed a method for building extraction 
from airborne oblique images by making use of facade texture. The method conducted the simultaneous processing of 2D image data and 3D points data generated from the same image set. Robust facade detection was done by utilizing horizontal and vertical facade texture together with height gradient from 3D points.

Nex et al. (2013) presented a methodology for the automated extraction of building footprints from photogrammetric point clouds. Points laying on walls with horizontal normal vectors were used to extract building boundaries, which can be bridged and smoothed to construct building footprints.

Rau et al. (2015) proposed a method to automatically classify photogrammetric point clouds into various urban object classes. A rule-based hierarchical semantic classification scheme that utilizes spectral information and geometry and topology related features was developed, in which the object height and gradient features were derived from the photogrammetric point clouds to assist in the detection of elevated objects, particularly for the roof and facade.

Verdie et al. (2015) introduced a solution to detect buildings from 3D surface meshes generated by multi-view stereo systems. In a pre-processing step, this method over-segment the input mesh into superfacets, which are obtained by clustering the triangle facets with similar shape operator matrices. From the geometric attributes computed per superfacet, a Markov Random Field is used to label each superfacet with one of the four classes: ground, tree, facade and roof.

\subsubsection{Building Reconstruction from Oblique Imagery}

The research of $3 \mathrm{D}$ building reconstruction from oblique airborne imagery has been increasingly focused. Some researchers have reviewed recent works of building reconstruction from oblique airborne imagery (Musialski, 2012; Wang,2013). Based on photogrammetric point clouds or meshes, most researches concentrate on how to simplify raw data and obtain semantized and compact 3D building models. Chauve et al. (2010) proposed a method for automatic simplified piecewise-planar 3D building reconstruction and completion from massive and noisy photogrammetric point clouds. The major contribution of this work is an adaptive decomposition of 3D space induced by planar primitives, namely a polyhedral cell complex.

Lafarge et al. (2012) presented a robust method for modeling buildings from Multi-View Stereo imagery. This method guarantees a high generalization level while having semantized and compact representations. Geometric 3D-primitives such as planes, cylinders, spheres describe regular roof sections, and are combined with mesh-patches that represent irregular roof components.

Xiong et al. (2014) proposed a parameter-free algorithm to robustly derive roof structures from photogrammetric point clouds. The point cloud was segmented into planar roof patches and the points connecting the patches were searched for and grouped as structure points and boundaries. The roof models were achieved by sequential connecting all the boundary lines and projecting the outer boundaries onto ground as walls.

Verdie et al. (2015) presented an approach that reconstructs 3D buildings in the form of levels of detail (LODs) from surface meshes generated by multi-view stereo systems. This method implemented a process for the global regularization of planes with a hierarchical organization of geometric relationships. And a min-cut formulation applied to a discrete approximation of a 3D planar arrangement for robust reconstruction.

Salinas et al. (2015) proposed a structure-aware mesh decimation method that mainly used to simplify dense surface meshes of complex buildings in urban scene. This approach takes as input a surface triangle mesh and a set of planar proxies pre-detected, and generates as output a simplified mesh where coarse-scale structures are preserved via an error metric and specific rules.

\section{METHODOLOGY}

We apply our method to both $2 \mathrm{~d}$ oblique imagery $3 \mathrm{~d}$ mesh derived from multi-view imagery. First, a contour-based method was employed to quickly detect buildings from raw meshes. Second, in order to preserve sharp edges in mesh segmentation, feature lines can be extracted from building meshes and added as constraints in the hierarchical face clustering. Third, to represent the topology structure of buildings, straight skeleton can be generated through the intersection of adjacent segmented planes, and optimized by defined regularities. Moreover, straight line segments extracted from multi-view oblique images can be used to refine $2 \mathrm{~d}$ straight skeleton that is back projected from $3 \mathrm{~d}$ straight skeleton. Finally, $3 \mathrm{~d}$ building models containing complete roofs and dominant facades can be constructed with refined topology structures.

\subsection{Contour-based Building Detection}

We employ a fast and robust method for building detection from urban meshes. This method is based on contour clustering of meshes (Zhang et al. 2008). As the building mesh has detailed information at facades, it's appropriate to use contour lines to describe $3 \mathrm{~d}$ building structures.

The first stage of building detection is to extract the contour lines from mesh data. We slice the mesh at a selected vertical interval through the intersection of layered horizontal planes and mesh. Therefore, every slice layer containing several closed contour lines can be extracted to represent shape abstractions of 3D objects, and it's necessary to determine which of these contours specify individual models. Therefore, one step of contour correspondence is adopted to find the correct connections between the contours of adjacent slices. The overlapping area of two regions enclosed by the two contours is used to estimate the contour correspondence parameter $\mathrm{T}$.

$$
\mathrm{T}=\max \left\{\frac{M}{S 1}, \frac{M}{S 2}\right\}
$$

where $\mathrm{S} 1$ and $\mathrm{S} 2$ indicate areas of contour $\mathrm{P} 1$ and $\mathrm{P} 2$ at adjacent slices, and $\mathrm{M}$ is the overlapping area. When $\mathrm{T}>0.8$, this contour pair is set to be connected.

After the contour correspondence, all contours will be grouped to different clusters that represent individual objects. For different objects, their contours are usually different in their characteristics of clustering. The contours of a building are dense, regular and similar enough to form a cluster, while contours of trees may be also dense, but their shape is less regular, less similar and the area is smaller than that of buildings. With area, shape regularity and similarity factors, contour clusters of buildings can be distinguished.

However, when the mesh is generated with adjacent buildings and trees, extracted contours are always mixed that making building detection more difficult. In order to separate them, it's necessary to do the shape decomposition of contours. Firstly, for each contour cluster, we can estimate its main directions by the principal component analysis of contours, which has been simplified by Douglas-Peucker method. Secondly, all contours are projected to $x-y$ plane, in which contours at facades are always close to each other that indicate high density of projected contour lines. Then multiple line segments along the main directions can be extracted with dense projected contours and used to decompose original contours. Finally, contours containing both buildings and trees can be separated to form new contour clusters. 


\subsection{Feature Preserving Mesh Segmentation}

\subsubsection{Ridge-Valley Lines Extraction}

We start by detecting feature lines on mesh surfaces. In this step, we follow the method of [Ohtake et al. 2004] to extract the feature curves. In this work, compactly supported radial basis functions (CS-RBFs) is employed for global smoothing purposes. We approximate a triangle mesh by a CS-RBF surface and project the mesh vertices onto the surface. The curvature tensor and derivatives are estimated to detect curvature maxima and minima on mesh edges, which are used to trace ridges and valleys. Finally the extracted ridges and valleys are smoothed to remove the noise and obtain smooth curves. We measure the strength $\mathrm{T}$ of a feature line by combining line length and the integral of $\mathrm{k}_{\max }$ along the line, and ignore those feature lines for which the strength $\mathrm{T}$ is less than a user-specified value of threshold. Figure 1 shows the curvature max and detected feature lines.
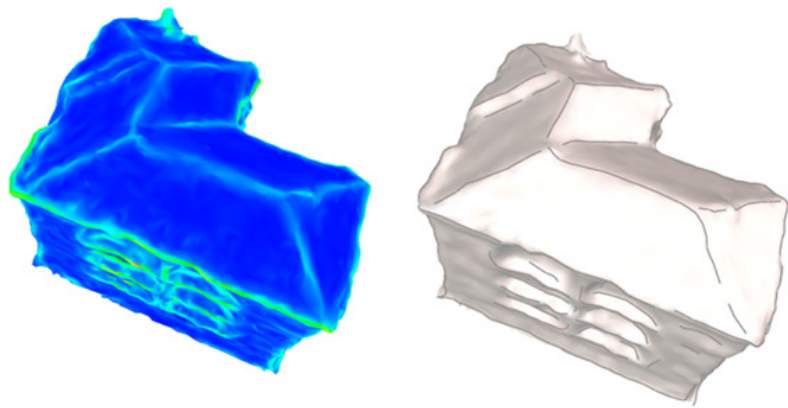

Fig.1. Left: curvature max. Right: smoothed feature lines

\subsubsection{Mesh Segmentation}

In fact, the derived ridges and valleys are curved lines, while feature lines of buildings are always straight. What's more, most of the ridge and valley curves are discretely distributed on the surfaces, and it is difficult to connect them to form a valid feature network. Therefore, it's necessary to straighten feature lines and construct the topology relationship of feature lines. Before mesh segmentation, feature lines, which have been weighted by their strength, are inserted into mesh to construct new feature line-constrained mesh.

Our work is based on Hierarchical Face Clustering (HFC) method (Garland et al. 2001). This method presented an efficient clustering algorithm which partitions a given surface into a hierarchy of disjoint face clusters. To construct a face hierarchy, it begin by forming the dual graph of the surface. The dual graph is defined by mapping every face of the surface to a node, and connecting two dual nodes by an edge if the corresponding faces are adjacent on the surface. As in figure 2, dual edge contraction corresponds to merging two adjacent face clusters into a single cluster.

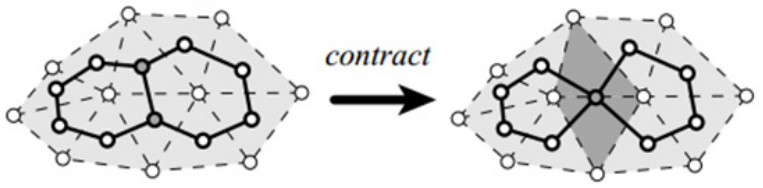

Fig.2. Dual edge contraction

Each dual edge is assigned a "cost" of contraction, and the system iteratively contracts the dual edge of least cost. Dual Quadric Metric is used to evaluate the cost of a dual contraction. Given a set of vertices $\left\{\mathrm{v}_{i}\right\}$, we define the dual quadric as

$$
\begin{gathered}
\mathrm{P}_{i}=\left(A_{i}, b_{i}, c_{i}\right)=\left(v_{i} v_{i}{ }^{T}, v_{i}, 1\right) \\
\mathrm{P}_{i}(n, d)=n^{T} A_{i} n+2 b_{i}{ }^{T}(d n)+c_{i} d^{2}
\end{gathered}
$$

so that we can evaluate this error using a corresponding set of quadrics:

$$
\mathrm{E}_{f i t}=\frac{1}{k} \sum_{i} P_{i}(n, d)=\frac{1}{k}\left(\sum_{i} P_{i}\right)(n, d)
$$

Two additional metrics are considered to construct the face hierarchy, which are orientation bias $\left(\mathrm{E}_{d i r}\right)$ and compact shape bias $\left(\mathrm{E}_{\text {shape }}\right)$. Therefore, the cost of a dual contraction will be determined by the combined error metric.

In our work, we propose a new error metric $\mathrm{E}_{\text {sharp }}$ to preserve feature lines during face clustering. If a large part of edges to be contracted are feature lines, the value of $\mathrm{E}_{\text {sharp }}$ is large that dual contraction should be prevented. What's more, as feature lines have provided shape constraints, the shape bias is disabled and then replaced by feature line constraint.

$$
\begin{gathered}
\mathrm{E}=\mathrm{E}_{\text {fit }}+\alpha_{1} \mathrm{E}_{\text {dir }}+\alpha_{2} \mathrm{E}_{\text {sharp }} \\
\mathrm{E}_{\text {sharp }}=\omega \frac{L_{\text {constrain }}}{L_{\text {contract }}}
\end{gathered}
$$

Where $\omega$ and $\mathrm{L}_{\text {constrain }}$ are the strength and length of feature lines to be contracted, $\mathrm{L}_{\text {contract }}$ is the total length of edges to be contracted, $\alpha_{1}$ and $\alpha_{2}$ are constants defined by the user.

After building the hierarchical tree, the segmentation result can be obtained by the number of clusters or defined maximum cost of edge contraction. Figure 3 shows the segmented result of VSA (Cohen et al. 2004) and our method. We compare 100 clusters by VSA with 50 clusters by our method, and it's obvious that our feature line-constrained segmentation preserve more sharp edges with less clusters.
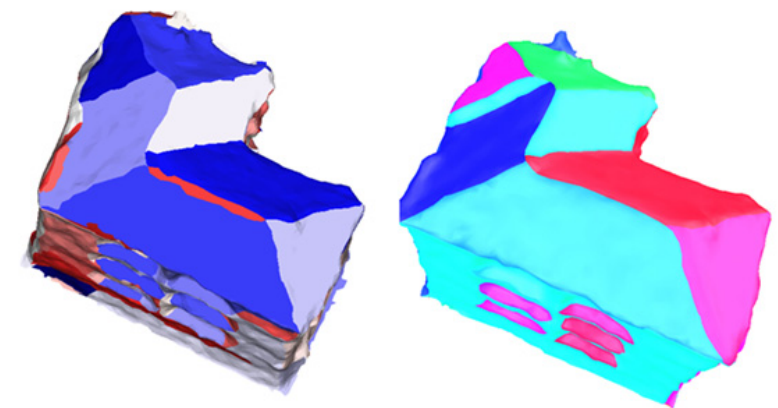

Fig.3. Left: 100 clusters by VSA. Right: 50 clusters by our method

\subsection{Topology Construction and Refinement}

\subsubsection{Topology Construction}

After mesh segmentation, planar patches have been detected. First, we should extract dominant facade planes, for the reason that each façade has a dominant and vertical plane as background for more detail modeling, such as window and balcony reconstruction, which are not considered in this paper. Nearly vertical planes are chosen as candidates for vertical plane fitting. Next, based on the dual graph of face clustering, we can easily build region adjacency graph of all planar regions. The intersections of two adjacent planes will generate new straight feature lines instead of original curved lines. Cycles of region adjacent graph indicate structure points, which connect different feature lines to form the straight and connected skeleton of buildings.

\subsubsection{Topology Refinement by regularities}

However, because of the noisy data and plane fitting errors, the constructed skeleton is not so regular and accurate that need to be optimized. Inspired by GlobFit [Li et al. 2011] and global regularities [Zhou et al. 2012], we mainly apply certain global regularities to refine feature lines. Various predefined regularities, such as parallelism, orthogonality, symmetry, height-equality, are employed to build geometric relationships for all feature lines. Figure 4 shows the result of topology refinement by these regularities. 
- Parallelism: lines in the set of $\{\mathrm{P} 1, \mathrm{P} 2, \mathrm{P} 3\},\{\mathrm{P} 4, \mathrm{P} 7\}$ are parallel with each other.

- Orthogonality: lines in the set of $\{\mathrm{P} 1, \mathrm{P} 2, \mathrm{P} 3\}$ are orthogonal with lines in the set of $\{\mathrm{P} 4, \mathrm{P} 7\}$.

- Symmetry: P2 is symmetric with P3 by P1, P5 is also symmetric with P6 by P1.

- Height-equality: horizontal lines P2,P3,P4 have equal height.

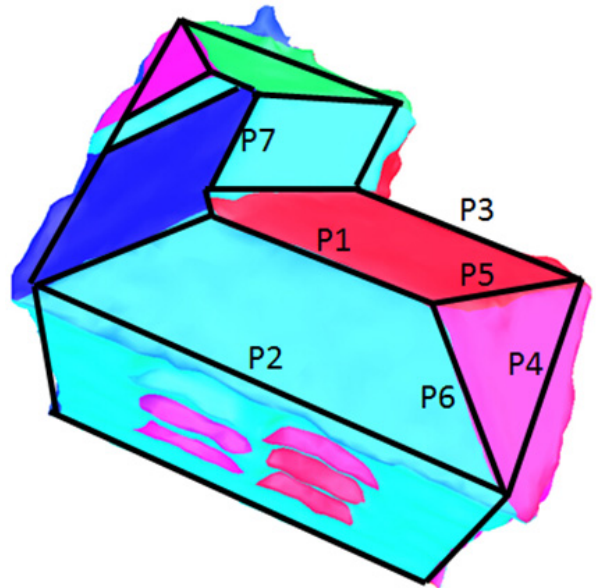

Fig.4. Straight skeleton refined by regularities

\subsubsection{Topology Refinement by multi-view imagery}

The poor quality of dense matching at the boundary of buildings makes feature lines inaccurate. In particular, when the roof has overhangs, eaves were removed during the intersection between roof planes and facade planes. In addition, the derived façade planes are always unreliable due to the noisy façade mesh, so that vertical feature lines generated by the intersection of adjacent façade planes are also inaccurate.

In order to solve these problems and obtain reliable 3D model structure, 2D feature lines of oblique airborne imagery are introduced to assist the topology refinement. The fast line segment detector (LSD) [Gioi et al. 2010] was applied to the oblique imagery to derive most of the straight line segments. As in figure 5(a), most line segments of roof boundaries and inner feature lines can be detected from nadir view image.

With depth images, the positions of the 3D line segments are translated to the image coordinate using depth value. Therefore, the straight 3D skeleton can be back projected to the corresponding oblique imagery. Figure 5(b) shows the back projection result of $3 \mathrm{D}$ feature lines. It can be found that feature lines projected at roof ridges and valleys fit well with image line segments, for the reason of good quality of image matching result at inner regions and our feature line-constrained mesh segmentation. Contrary to this good fitting, most feature lines projected at roof boundaries are far away with reliable boundaries detected from the image. This bad fitting result is caused by several reasons including the low quality of image matching at roof boundaries, noisy façade planes and roof overhangs.

Therefore, the main objective in our topology refinement is to rectify boundaries of roofs and façades. A line segments filtering step is firstly adopted to extract potential boundaries. With the distance and angle thresholds, line segments nearly parallel or orthogonal with boundary lines can be detected and used as candidate lines in the next refinement step. Figure 5(c) shows the filter result of chosen candidate lines. In addition, there are also missing boundary lines that hinder the structure refinement.

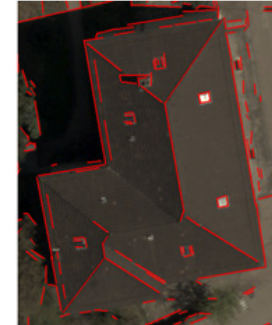

(a)

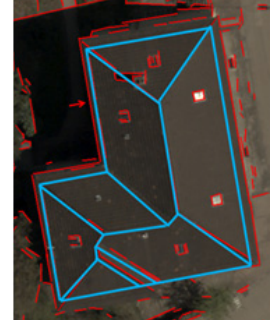

(b)

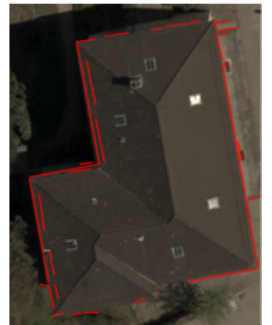

(c)
Fig.5. (a) LSD result with red lines. (b) blue lines indicate back-projected lines of 3D straight skeleton. (c) the filter result of chosen candidate lines

In fact, 2D line segments derived from multi-view images are different with each other, making it possible to combine them to form the complete candidate lines. As in figure 6, the filtered line segments differ in multiple oblique images and supplement each other. Based on the complete candidate lines, each boundary line is assigned with several corresponding candidate lines.
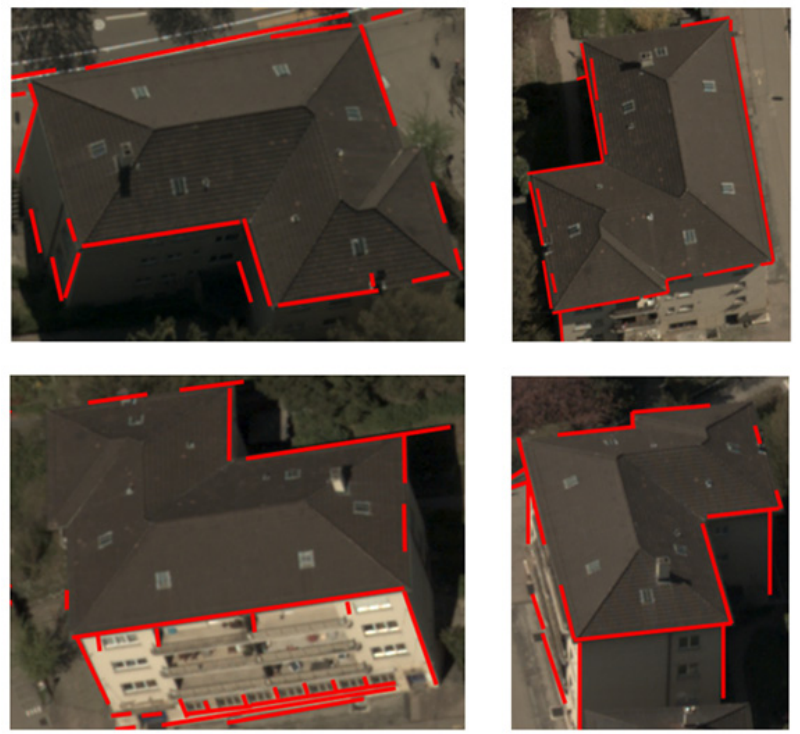

Fig.6. Different candidate lines of multi-view images

To solve the problem of how to determine the new positions of boundary lines, we take two operations to transform boundary lines: translation and decomposition. In general, translation is the major operation that each boundary line has different translations to its nearly parallel candidate lines. During the translation, the same parts of two lines can be calculated and then used to determine the decomposition operation. As in figure 7(a), both two cases contain two translations from Line L to L1 and L2, but their corresponding line parts are different. The left is the case that two corresponding parts P1 and P2 include each other, while the right case shows that P1 and P2 are connected or away from each other, but constitute the approximate shape of line L, which is promising to need the line decomposition. To make the further judgment of the right case, we also search orthogonal candidate lines to find the short line L3 between L1 and L2. If L3 found, line L will be decomposed at the position of L3, otherwise, the dividing position is determined by the midpoint of two neighbor end points. Figure 7(b) shows that the projected boundary line al should be decomposed into four line segments b1,b2,b3,b4, while orthogonal lines $\mathrm{c} 1, \mathrm{c} 2, \mathrm{c} 3$ are added to connect translated lines. 


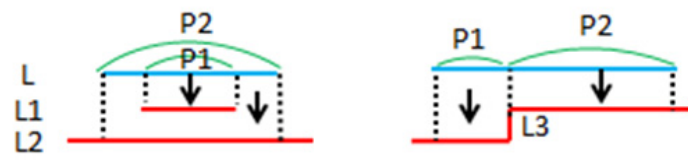

(a)

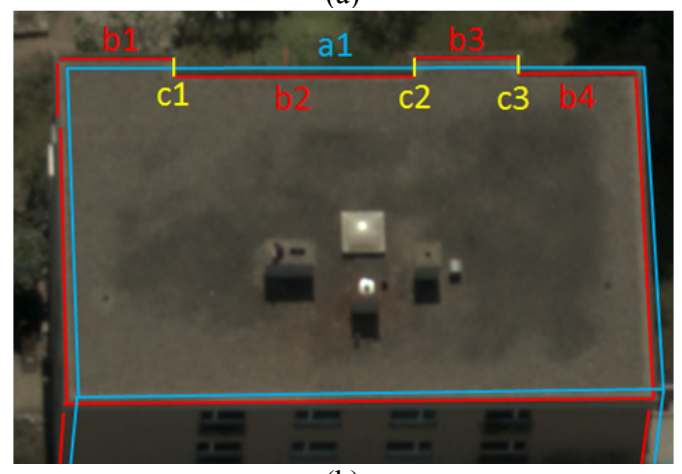

(b)

Fig.7. (a) Two operations to transform boundary lines. (b) an example of boundary line decomposition

If $2 \mathrm{D}$ boundary lines are transformed, their transformations in 3D should be realized. In general, the boundary line can be moved along its orthogonal direction on its roof plane, and the distance to be translated is calculated by the projected distance between 2D translated lines. What's more, these 3D boundary lines should be transformed under the constraints of their multiple associated regularities. For instance, boundary lines always have height-equality regularity that should be preserved during the transformation.

\section{EXPERIMENTAL RESULT}

The test dataset in the city of Zürich is provided by ISPRS/EuroSDR initiative Benchmark on High Density Image Matching for DSM Computation. In our experiment, 135 oblique aerial images captured at a GSD of $6 \mathrm{~cm}$ by a Leica RCD30 Oblique Penta camera are used.

Before our experiment, the software Agisoft Photoscan was used to generate 3D surface meshes. At first, all images were aligned by sparse matching. As in figure 8, the large-scale tie points were produced. Then we choose a small region (red box in figure 8) that consists of various types of buildings as the test area, which was used to build dense point cloud and 3D surface mesh. Figure 9(a) shows the textured mesh of this test area. Through the contour-based building detection, most buildings have been extracted as in figure 9(b), while a small building (red circle in figure 9(a)) missed due to its flat roof and low height façade.

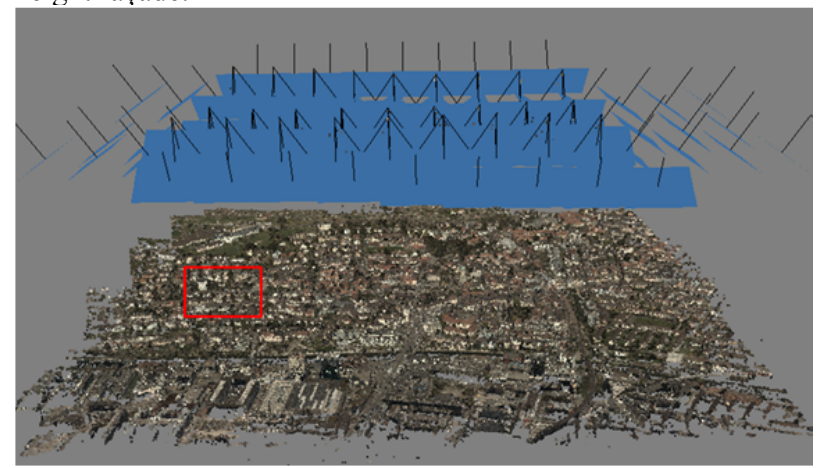

Fig.8.The result of tie points generated by Photoscan

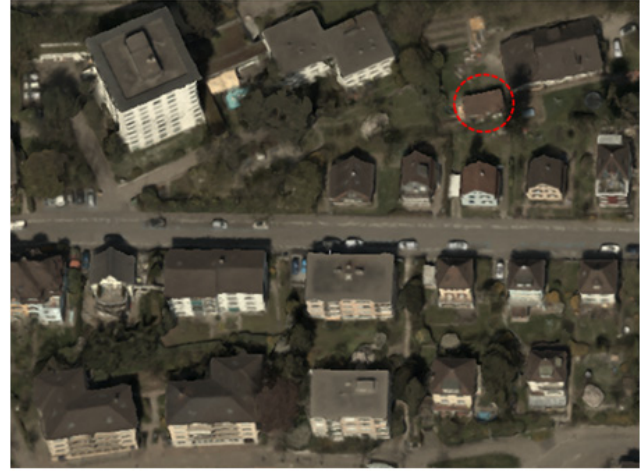

(a)
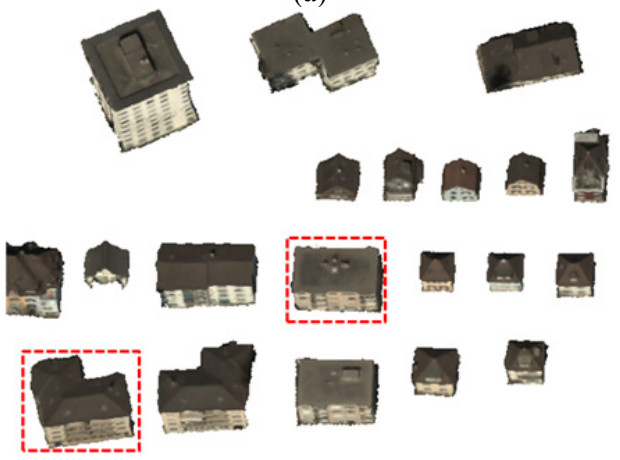

(b)

Fig.9. (a) 3D textured mesh of test area. (b) building meshes extracted by contour based method

Figure 10 shows two representative building models (red boxes in figure $9(\mathrm{~b})$ ). In figure 10(a), the building with flat roof has little shape shifting in the upper boundary, which could be easily eliminated by coarse plane intersection. With the refinement of feature lines in oblique images, small structures in the upper boundary can be recovered, while other boundaries are also rectified under the constraints of regularities. Figure 10(b) shows a building model with complex roof structure that fit well with the oblique image. On one hand, the accurate inner structure lines are formed by the feature preserving mesh segmentation and the refinement of regularities. And on the other hand, the accuracy of outer boundaries is mainly improved by the refinement of multi-view oblique images. Figure 10(c) shows that roof overhangs colored by blue dotted lines have been successfully reconstructed.
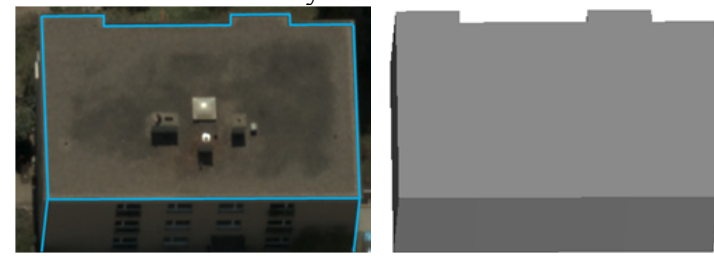

(a)
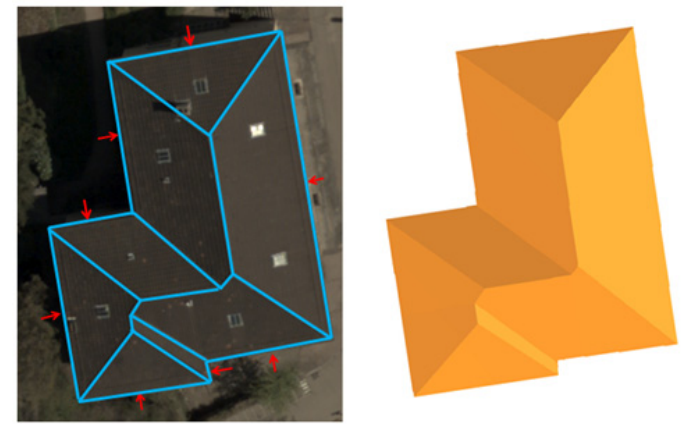

(b) 

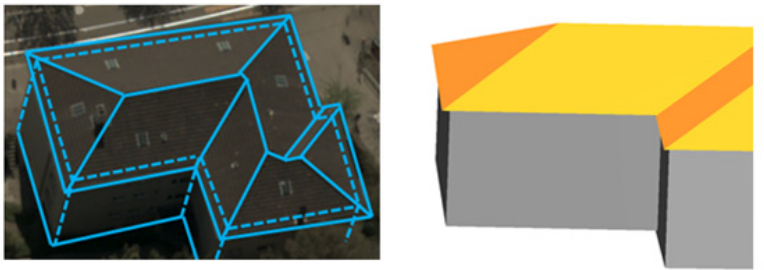

(c)

Fig.10. (a) The refined model with flat roof. (b) the refined model with complex roof structure. (c) generated roof overhangs of the building in (b).

\section{CONCLUSIONS}

This paper presents a feature line based method to detect and reconstruct 3D building models from oblique airborne imagery. Through the contour clustering method, buildings can be quickly and robustly detected from meshes. With the constraint of curved lines derived from meshes, the feature preserving mesh segmentation method is applied to accurately extract 3D straight skeleton, which could be optimized by our defined regularities. What's more, straight feature lines derived from multi-view images are used to rectify inaccurate parts of $3 \mathrm{D}$ straight skeleton of buildings, especially at boundaries of roofs and facades. Therefore, low quality model can be refined by the accuracy improvement of mesh feature lines and rectification with feature lines of multi-view images. The experiments reveal that the proposed method can obtain convincing results and promote the quality of 3D building models in urban scene.

\section{ACKNOWLEDGEMENT}

This work was supported by the National Basic Research Program (973 Program) of China under Grant 2012CB719904.

\section{REFERENCE}

Chauve, A. L., Labatut, P., \& Pons, J. P. 2010. Robust piecewise-planar 3D reconstruction and completion from large-scale unstructured point data. In Computer Vision and Pattern Recognition (CVPR). pp. 1261-1268.

Chen L C, Teo T A, Shao Y C, et al. 2004. Fusion of LIDAR data and optical imagery for building modeling. International Archives of Photogrammetry and Remote Sensing, 35(B4): $732-737$.

Cheng, L., Gong, J., Li, M., \& Liu, Y. (2011). 3D building model reconstruction from multi-view aerial imagery and lidar data. Photogrammetric Engineering \& Remote Sensing, 77(2), 125-139.

Cohen-Steiner, D., Alliez, P., \& Desbrun, M. 2004. Variational shape approximation. In ACM Transactions on Graphics (TOG) (Vol. 23, No. 3, pp. 905-914). ACM.

David Salinas, Florent Lafarge, Pierre Alliez. 2015. Structure-Aware Mesh Decimation. Computer Graphics Forum, Wiley-Blackwell, pp.20.

Garland M, Willmott A, Heckbert P S. 2001. Hierarchical face clustering on polygonal surfaces, Proceedings of the 2001 symposium on Interactive 3D graphics. ACM,: 49-58.

Haala, N., and M. Kada, 2010. An update on automatic 3D building reconstruction, ISPRS Journal of Photogrammetry and Remote Sensing, 65:570-580

Lafarge, F., \& Mallet, C. (2012). Creating large-scale city models from 3D-point clouds: a robust approach with hybrid representation. International journal of computer vision, 99(1), 69-85.

Li, Y., Wu, X., Chrysathou, Y., Sharf, A., Cohen-Or, D., \& Mitra N. J. 2011. Globfit: Consistently fitting primitives by discovering global relations. In ACM Transactions on Graphics (TOG) (Vol. 30, No. 4, p. 52).

Musialski P, Wonka P, Aliaga D G, et al. 2013. A survey of urban reconstruction, Computer Graphics Forum. 32(6): 146-177.

Nex, F., Rupnik, E., \& Remondino, F. (2013). Building footprints extraction from oblique imagery. ISPRS Ann. Photogramm. Remote Sens. Spat. Inf. Sci, 2, 61-66.

Ohtake, Y., Belyaev, A., \& Seidel, H. P. 2004. Ridge-valley lines on meshes via implicit surface fitting. In ACM Transactions on Graphics (TOG) (Vol. 23, No. 3, pp. 609-612).

Rau J Y, Jhan J P, Hsu Y C. 2015. Analysis of Oblique Aerial Images for Land Cover and Point Cloud Classification in an Urban Environment. IEEE Transactions on Geoscience and Remote Sensing, vol. 53, issue 3, pp. 1304-1319.

Von Gioi, R. G., Jakubowicz, J., Morel, J. M., \& Randall, G. (2012). LSD: a line segment detector. Image Processing On Line, 2(3), 5.

Wang, R. (2013). 3D building modeling using images and LiDAR: A review. International Journal of Image and Data Fusion, 4(4), 273-292.

Xiao, J., Gerke, M., \& Vosselman, G. (2012). Building extraction from oblique airborne imagery based on robust façade detection. ISPRS journal of photogrammetry and remote sensing, 68, 56-68.

Xiong, B., Elberink, S. O., \& Vosselman, G. (2014). Building modeling from noisy photogrammetric point clouds. ISPRS Annals of Photogrammetry, Remote Sensing and Spatial Information Sciences, 1, 197-204

Yannick Verdie, Florent Lafarge, Pierre Alliez. 2015. LOD Generation for Urban Scenes. ACM Transactions on Graphics, Association for Computing Machinery .

Zhang, J., Li, L., Lu, Q., \& Jiang, W. 2008. Contour Clustering Analysis for Building Reconstruction from LIDAR Data. In Proceedings of The XXI Congress the international society for Photogrammetry and Remote Sensing (pp. 355-360).

Zhang, W., Wang, H., Chen, Y., Yan, K., \& Chen, M. (2014). 3D Building Roof Modeling by Optimizing Primitive's Parameters Using Constraints from LiDAR Data and Aerial Imagery. Remote Sensing, 6(9), 8107-8133.

Zhou, Q. Y., \& Neumann, U. (2012). 2.5 D building modeling by discovering global regularities. In Computer Vision and Pattern Recognition (CVPR), 2012 IEEE Conference on (pp. 326-333). IEEE. 